\title{
Lifting group actions, equivariant towers and subgroups of non-positively curved groups
}

\author{
RICHARD GAELAN HANLON \\ EdUARDo MARTÍNEZ-PEDROZA
}

\begin{abstract}
If $\mathcal{C}$ is a class of complexes closed under taking full subcomplexes and covers and $\mathcal{G}$ is the class of groups admitting proper and cocompact actions on one-connected complexes in $\mathcal{C}$, then $\mathcal{G}$ is closed under taking finitely presented subgroups. As a consequence the following classes of groups are closed under taking finitely presented subgroups: groups acting geometrically on regular CAT(0) simplicial complexes of dimension $3, k$-systolic groups for $k \geq 6$, and groups acting geometrically on 2 -dimensional negatively curved complexes. We also show that there is a finite non-positively curved cubical 3-complex that is not homotopy equivalent to a finite non-positively curved regular simplicial 3-complex. We include applications to relatively hyperbolic groups and diagrammatically reducible groups. The main result is obtained by developing a notion of equivariant towers, which is of independent interest.
\end{abstract}

20F67; 57M07

\section{Introduction}

We show that some classes of non-positively curved groups are closed under taking finitely presented subgroups. We assume all spaces are combinatorial complexes and all maps are combinatorial; see Definition 3.1. A complex is one-connected if it is connected and simply connected. A subcomplex $K$ of $X$ is full if for any cell $\sigma \subset X, \partial \sigma \subset K$ implies $\sigma \subset K$. An action of a group $G$ on a space $X$ is proper if for all compact subsets $K$ of $X$ there are finitely many group elements $g$ such that $K \cap g(K) \neq \varnothing$. The action is cocompact if there is a compact subset $K$ of $X$ such that the collection $\{g K: g \in G\}$ covers $X$. Our main result is the following.

Theorem 1.1 Let $\mathcal{C}$ be a category of complexes closed under taking full subcomplexes and topological covers. Let $\mathcal{G}$ be the category of groups acting properly and cocompactly by combinatorial automorphisms on one-connected complexes in $\mathcal{C}$. Then $\mathcal{G}$ is closed under taking finitely presented subgroups. 
Some words about the literature. A theorem of Steve Gersten states that finitely presented subgroups of hyperbolic groups of cohomological dimension at most 2 are hyperbolic [8]. This is a result only for dimension 2, since Noel Brady exhibited a 3-dimensional compact locally CAT(0) cube complex with hyperbolic fundamental group containing a finitely presented subgroup that is not hyperbolic [2]. Another result on the positive side is that finitely presented subgroups of torsion-free systolic groups are systolic; this was proved by Daniel Wise [24]. The proof of Theorem 1.1 builds on ideas of Bridson and Wise [4], Gersten [8], Howie [12] and Wise [24], and is self-contained in the paper.

\subsection{Sample applications}

A regular simplicial complex is a piecewise Euclidean simplicial complex where each 1-cell has unit length. A result of Rena Levitt shows that the category of regular locally CAT(0) simplicial complexes of dimension 3 is closed under taking full subcomplexes [16]. It is immediate that this category is closed under taking covers. Recall that a group action on a metric space is said to be geometric if it is proper, cocompact and by isometries.

Corollary 1.2 If $G$ acts geometrically on a regular CAT(0) simplicial complex of dimension 3, then any finitely presented subgroup acts geometrically on a regular CAT(0) simplicial complex of dimension 3.

It is not known whether Corollary 1.2 holds for higher dimensions. However, the proof presented here does not generalize since Levitt has exhibited regular locally CAT(0) simplicial complexes of dimension at least 4 containing full subcomplexes that are not locally CAT(0).

In [2], Noel Brady constructed a compact 3-dimensional locally CAT(0) cubical complex $X$ such that $\pi_{1}(X)$ contains a finitely presented subgroup that does not admit a finite classifying space. Since every compact locally CAT(0) space is a classifying space for its fundamental group, the previous corollary together with Brady's example implies the following statement.

Corollary 1.3 There is a 3-dimensional finite locally CAT(0) cubical complex that is not homotopy equivalent to a 3 -dimensional finite regular locally CAT(0) simplicial complex.

Levitt proved that non-positively regular CAT(0) simplicial complexes of dimension 3 are systolic complexes [17]; for this larger class our main theorem also applies. 
The notion of simplicial non-positive curvature for simplicial complexes was introduced by Januszkiewicz and Świątkowski in [15] and independently by Haglund [10] as a combinatorial analog of non-positive curvature metric conditions. A simplicial complex $L$ is flag if any set of vertices that are pairwise connected by $1-$ cells of $L$ spans a simplex in $L$. A simplicial complex $L$ is $k$-large, $k \geq 6$, if $L$ is flag and there are no embedded cycles of length less than $k$ that are full subcomplexes of $L$. A simplicial complex $X$ is locally $k$-large if the links of all simplices in $X$ are $k$-large. The fact that the category of locally $k$-large simplicial complexes is closed under taking covers and full subcomplexes immediately follows from the definitions. A group is $k$-systolic if it acts properly and cocompactly by simplicial automorphisms on a one-connected locally $k$-large simplicial complex. Wise proved that finitely presented subgroups of torsion free $k$-systolic groups are $k$-systolic using a tower argument [24]. Corollary 1.4 extends Wise's result to include groups with torsion. Previously to this work, the statement of Corollary 1.4 was also proved by Gašper Zadnik using different methods [26].

Corollary 1.4 For $k \geq 6$, if $G$ is a $k$-systolic group then any finitely presented subgroup of $G$ is $k$-systolic.

In [20], Osajda introduced the notion of complexes with $S D_{2}^{*}(k)$ links for $k \geq 6$. There it is proved that the class of fundamental groups of compact complexes with $S D_{2}^{*}(k)$ links is closed under taking finitely presented subgroups [20, Theorem 8.7]. His proof is a tower argument for compact complexes, and in particular it is shown that $S D_{2}^{*}(k)$ complexes are closed under taking covers and full subcomplexes. It follows that Theorem 1.1 applies to the class of $S D_{2}^{*}(k)$ providing an extension of Osajda's result.

A 2-complex $X$ is negatively curved if it satisfies one of the following conditions:

(1) (Metric condition) There is $\kappa<0$ such that $X$ admits the structure of a locally $\mathrm{CAT}(\kappa) M_{\kappa}$-complex.

(2) (Conformal condition) There is an assignment of a non-negative real number, called an angle, to each corner of each 2-cell such that the sum of the angles on an $n$-gon is strictly less than $(n-2) \pi$ and links of 0 -cells satisfy Gromov's link condition: Every non-trivial circuit in the link is of angular measure at least $2 \pi$.

A group acting geometrically on a one-connected negatively curved 2-complex is word hyperbolic (Bridson and Haefliger [3] and Gromov [9]). Gersten proved that finitely presented subgroups of fundamental groups of finite negatively curved 2-complexes are word hyperbolic [8, Theorem 2.1]. One easily verifies that the category of negatively curved 2-complexes is closed under taking subcomplexes and covers. The following corollary extends Gersten's result. 
Corollary 1.5 (Subgroups of 2-dimensional negatively curved groups) Let $Y$ be a one-connected negatively curved proper and cocompact $H-2$-complex. If $G \leq H$ is finitely presented, then $G$ is word hyperbolic.

We remark that Corollary 1.5 would follow from Gersten's result if $H$ were known to be virtually torsion-free. This is an open question for hyperbolic groups [9]. We also obtain an analogous result for relatively hyperbolic groups stated below.

A $G$-complex $X$ is almost proper if $G$ acts properly on the complement of the zeroskeleton of $X$. Observe that a proper action is almost proper. A group is called slender if all its subgroups are finitely generated. For definitions of relatively hyperbolic groups and fine complexes we refer the reader to Section 5.2.

Theorem 1.6 (Theorem 5.15, subgroups of 2-dimensional relatively hyperbolic groups) Let $Y$ be a one-connected negatively curved, fine, almost proper and cocompact $H-2-$ complex such that $H$-stabilizers of cells are slender. If $G \leq H$ is finitely presented then $G$ is hyperbolic relative to a finite collection of $G$-stabilizers of cells of $Y$.

The term "diagrammatically reducible complex", defined below, was introduced by Gersten in connection with the study of equations over groups [7]. The notion was first used, with different names, by Chiswell, Collins and Huebschmann [5] and Sieradski [23]. Recall that an immersion is a locally injective map, and a near-immersion is a map that is locally injective except at 0 -cells of the domain. A 2-complex $X$ is diagrammatically reducible if there are no near-immersions $C \rightarrow X$, where $C$ is a cell structure for the 2-dimensional sphere.

Since the composition of a near-immersion followed by an immersion is a nearimmersion, the category of diagrammatically reducible complexes is closed under taking covers and subcomplexes. This category of complexes includes locally CAT(0) 2-complexes, certain classes of small cancellation complexes, conformal negatively curved 2-complexes, spines of hyperbolic knots, and non-positively curved square complexes, to name a few examples. Recall that a proper $G$-complex $X$ is a model for $\underline{E} G$ if for every finite subgroup $F \leq G$, the fixed point set $X^{F}$ is contractible. The following theorem is proved in Section 5.

Theorem 1.7 (Theorem 5.10, diagrammatically reduced groups) Let $X$ be a diagrammatically reducible one-connected proper $H-2$-complex. If $G \leq H$ is finitely presented, then $G$ admits a diagrammatically reducible 2-dimensional cocompact model for $\underline{E} G$. 


\subsection{Equivariant towers and the proof of Theorem 1.1}

Briefly, Theorem 1.1 is proved using an extension of the tower method to equivariant maps. There are several applications of towers in combinatorial group theory as illustrated in Gersten [8], Howie [12; 13], Hruska and Wise [14], Wise [24; 25] and the results of this paper. Towers are a geometric technique from 3-manifold topology introduced by Papakyriakopolous [21], and later brought to combinatorial group theory by Howie [13]. The idea of using towers to investigate finitely presented subgroups as used in this article goes back to Gersten [8] and Shalem [4]. A combinatorial map $X \rightarrow Y$ between connected CW-complexes is a tower if it can be expressed as a composition of inclusions and covering maps. A tower lifting $f^{\prime}$ of $f$ is a factorization $f=g \circ f^{\prime}$, where $g$ is a tower. The lifting $f^{\prime}$ is trivial if $g$ is an isomorphism and the lifting is maximal if the only tower lifting of $f^{\prime}$ is the trivial one. It is well known that if $X$ is a finite complex, then any combinatorial map $X \rightarrow Y$ admits a maximal tower [12, Lemma 3.1]. A tower is called an $\mathcal{F}$-tower if it is a composition of covering maps and inclusions of full subcomplexes.

By a locally finite complex, we mean a complex such that every closed cell intersects finitely many closed cells.

Theorem 1.8 (Theorem 3.14, maximal equivariant $\mathcal{F}$-towers) Let $f: X \rightarrow Y$ be a $G$-map. If $X$ is one-connected and $G$-cocompact and $Y$ is locally finite, then $f$ has a maximal $\mathcal{F}$-tower lifting $f=g \circ f^{\prime}$, where $g$ and $f^{\prime}$ are $G$-maps.

An analogous result to Theorem 1.8 where $Y$ is not required to be locally finite and instead $f^{\prime}$ is only a maximal tower lifting (not a maximal $\mathcal{F}$-tower lifting) also holds; see Theorem 3.18. This slightly different result is relevant to applications such as Theorem 1.6.

In [24], there is a result similar to Theorem 1.8 stating that if $X \rightarrow Y$ is a map between finite simplicial complexes then there is a maximal expanded tower lifting. This is a different class of towers, and neither result subsumes the other. The class of expanded towers works well in the setting of 2-skeletons of systolic complexes, which Wise used to prove Corollary 1.4 in the torsion-free case.

A consequence of Theorem 1.8 is the following.

Theorem 1.9 (Theorem 4.1, Existence of immersed cocompact $\mathcal{F}$-cores) If $Y$ is a one-connected, proper and locally finite $H$-complex and $G \leq H$ is finitely presented, then there is a one-connected cocompact $G$-complex $X$ and a $G$-equivariant $\mathcal{F}$-tower $X \rightarrow Y$. 
The proof of Theorem 1.9 relies on the construction of a one-connected and cocompact $G$-complex $X_{0}$ together with a $G$-map $f: X_{0} \rightarrow Y$; this construction uses the hypothesis that $Y$ is one-connected. Given such a map, since $X_{0}$ is one-connected, there is an equivariant maximal $\mathcal{F}$-tower lifting $f=g \circ f^{\prime}$ with $g: X \rightarrow Y$. The maximality of $f^{\prime}$ implies that $X$ is one-connected, and together with $Y$ being locally finite, that $X$ is $G$-cocompact.

We also prove a version of Theorem 1.9 without the assumption that $Y$ is locally finite, but with the weaker conclusion that the $G$-map $X \rightarrow Y$ is only a tower; see Theorem 4.4. The proof of our main result, Theorem 1.1, follows immediately from Theorem 1.9.

Proof of Theorem 1.1 Let $Y$ be a one-connected complex in $\mathcal{C}$, let $H$ be a group acting properly and cocompactly on $Y$, and let $G \leq H$ be a finitely presented subgroup. Since $Y$ is a proper and cocompact $H$-complex, it is locally finite. By Theorem 1.9, there is a one-connected cocompact $G$-complex $X$ and an $G$-equivariant $\mathcal{F}$-tower $X \rightarrow Y$. By equivariance, $X$ is also a proper $G$-complex. Since $\mathcal{C}$ is closed under taking full-subcomplexes and covers, it follows that $X$ is in $\mathcal{C}$. Therefore $G$ is in $\mathcal{G}$.

\subsection{Outline of the paper}

The rest of the paper is organized in four sections. Section 2 contains a result that provides sufficient conditions to lift a group action on a space to an intermediate cover. Section 3 contains the definition of equivariant towers and the proof of the existence of maximal equivariant $\mathcal{F}$-towers. Section 4 contains the proof of the existence of immersed cocompact $\mathcal{F}$-cores. The last section contains the proofs of Theorems 1.5 and 1.7 .

\section{Acknowledgments}

Thanks to Daniel Wise and Mark Sapir for both suggesting the applications to systolic complexes, and Daniel Wise for sharing his unpublished preprint [24], which motivated part of the work of this paper. We also thank Damian Osajda for useful comments. We also thank Saya Martínez-Aoki for assistance during this work. Last but not least, we thank the anonymous referee for feedback, several suggestions and corrections, and pointing out a serious mistake in an earlier version of the paper. We also acknowledge funding by the Natural Sciences and Engineering Research Council of Canada NSERC. 


\section{Lifting group actions}

In this section, all spaces are topological spaces that are path-connected, locally pathconnected and semilocally simply connected. These are the standard hypotheses for the existence of universal covers. All maps between spaces are continuous. For standard results on covering space theory we refer the reader to Hatcher's textbook on algebraic topology [11].

Definition 2.1 Let $X$ be a $G$-space and let $Y$ be an $H$-space. A map $f: X \rightarrow Y$ is equivariant with respect to a group homomorphism $f_{\#}: G \rightarrow H$ if $f(g . x)=f_{\#}(g) f(x)$ for every $g \in G$ and $x \in X$.

If $Y$ is an $H$-space then the universal cover $\tilde{Y}$ is naturally an $\tilde{H}$-space and the covering map $\tilde{Y} \rightarrow Y$ is equivariant with respect to a natural group homomorphism $\tilde{H} \rightarrow H$, as the theorem below states. The proof is patterned after an argument by Bridson and Haefliger in the context of complexes of groups [3, Chapter III.C 1.15].

Theorem 2.2 (Lifting an action to the universal cover) Let $Y$ be an $H$-space, and let $\rho: \tilde{Y} \rightarrow Y$ be the universal covering map. Then there is a group $\tilde{H}$ and action $\tilde{H} \times \tilde{Y} \rightarrow \tilde{Y}$ with the following properties.

(1) There is an exact sequence of groups

$$
1 \rightarrow \pi_{1} Y \rightarrow \tilde{H} \rightarrow H \rightarrow 1 .
$$

(2) The covering map $\tilde{Y} \rightarrow Y$ is equivariant with respect to $\tilde{H} \rightarrow H$.

(3) The restriction of $\tilde{H} \times \tilde{Y} \rightarrow \tilde{Y}$ to $\pi_{1} Y \times \tilde{Y} \rightarrow \tilde{Y}$ is the standard action by deck transformations of $\pi_{1} Y$ on $\tilde{Y}$.

(4) For each $\tilde{y} \in \tilde{Y}$ mapping to $y \in Y$, the homomorphism $\tilde{H} \rightarrow H$ restricts to an isomorphism $\widetilde{H}_{\tilde{y}} \rightarrow H_{y}$ between the $\widetilde{H}$-stabilizer of $\tilde{y}$ and the $H$-stabilizer of $y$.

(5) If $G \times \tilde{Y} \rightarrow \tilde{Y}$ is an action satisfying the four analogous properties above, then there is an isomorphism $\Phi: G \rightarrow \widetilde{H}$ such that $g \cdot \tilde{y}=\Phi(g) \cdot \tilde{y}$ for all $\tilde{y} \in \tilde{Y}$.

(6) If $f: X \rightarrow Y$ is equivariant with respect to $f_{\#}: G \rightarrow H$, and $X$ is one-connected, and $\tilde{f}: X \rightarrow \tilde{Y}$ is a lifting of $f$, then $\tilde{f}$ is equivariant with respect to a group homomorphism $\tilde{f}: G \rightarrow \widetilde{H}$ that lifts $f: G \rightarrow H$.

It is an immediate corollary that there exists liftings to intermediate covers that are $H$-regular as defined below. 
Definition 2.3 ( $H$-regular covers) Let $Y$ be an $H$-complex. A covering map $\hat{Y} \rightarrow Y$ is $H$-regular if the composition $\pi_{1} \hat{Y} \rightarrow \pi_{1} Y \rightarrow \widetilde{H}$ is a normal subgroup of $\tilde{H}$.

Remark 2.4 Let $Y$ be an $H$-complex. By definition, the universal cover of an $H-$ complex is $H$-regular. Furthermore any cover of $Y$ associated to a characteristic subgroup of $\pi_{1}(Y)$ is $H$-regular.

Corollary 2.5 (Lifting an action to an intermediate cover) Let $Y$ be an $H$-space and let $\hat{Y} \rightarrow Y$ be an $H$-regular cover. Then the quotient group $\hat{H}=\widetilde{H} / \pi_{1} \hat{Y}$ acts on $\hat{Y}$ and the map $\hat{Y} \rightarrow Y$ is equivariant with respect to $\hat{H} \rightarrow H$. Furthermore, stabilizers of points are preserved in the sense that if $\hat{y} \in \hat{Y}$ maps to $y \in Y$, then the restriction $\hat{H}_{\hat{y}} \rightarrow H_{y}$ is an isomorphism.

Proof of Theorem 2.2 Let $y_{0}$ be a point of $Y$, and recall that $\tilde{Y}$ can be identified with the set

$$
\tilde{Y}=\left\{[c] \mid c \text { is a path in } Y \text { starting at } y_{0}\right\},
$$

where $[c]$ denotes the homotopy class of $c$ with respect to homotopies fixing the endpoints $c(0)$ and $c(1)$. The covering map $\tilde{Y} \rightarrow Y$ is interpreted as sending $[c]$ to $c(1)$. The action of $\pi_{1}\left(Y, y_{0}\right)$ on $\widetilde{Y}$ is given by

$$
\pi_{1}\left(Y, y_{0}\right) \times \tilde{Y} \rightarrow \tilde{Y}, \quad[\gamma] \times[c] \mapsto[\gamma * c] .
$$

For details of this standard construction of the universal cover and the action of the fundamental group, we refer the reader to [11]. Let

$$
\widetilde{H}=\left\{(h,[c]) \mid h \in H, c \text { a path in } Y \text { from } y_{0} \text { to } h \cdot y_{0}\right\} .
$$

The group operation on $\widetilde{H}$ is given by

$$
\tilde{H} \times \tilde{H} \rightarrow \tilde{H}, \quad(h,[c]) \times\left(h^{\prime},\left[c^{\prime}\right]\right) \mapsto\left(h h^{\prime},\left[c * h \cdot c^{\prime}\right]\right),
$$

where as usual $*$ denotes concatenation of paths. Observe that the operation is well defined since for any pair of paths $f \in[c]$ and $f^{\prime} \in\left[c^{\prime}\right]$ the terminal point of $f$ equals the initial point of $h f^{\prime}, c * h . c^{\prime}$ is homotopic relative to endpoints to $f * h . f^{\prime}$, and the terminal point of $c * h . c^{\prime}$ is $h h^{\prime} \cdot y_{0}$. Showing that this operation endows $\tilde{H}$ with a group structure is routine and it is left to the interested reader. The action of $\widetilde{H}$ on $\tilde{Y}$ is given by

$$
\tilde{H} \times \tilde{Y} \rightarrow \tilde{Y}, \quad(h,[c]) \cdot\left[c^{\prime}\right]=\left[c * h \cdot c^{\prime}\right],
$$

and one easily verifies that it is a well-defined action. Now we verify the six properties. 
Properties (1), (2) and (3) Observe that the natural projection

$$
\widetilde{H} \rightarrow H, \quad(h,[c]) \mapsto h
$$

is a surjective homomorphism with kernel

$$
\left\{(1,[c]) \mid c \text { is a closed path with } c(0)=c(1)=y_{0}\right\} \cong \pi_{1}\left(Y, y_{0}\right) .
$$

By definition the action $\tilde{H} \times \tilde{Y} \rightarrow \tilde{Y}$ extends the action of $\pi_{1}\left(Y, y_{0}\right)$ on $\tilde{Y}$. To verify that $\tilde{Y} \rightarrow Y$ is equivariant with respect to $\widetilde{H} \rightarrow H$, let $\left(h^{\prime},\left[c^{\prime}\right]\right) \in \widetilde{H}$ and $[c] \in \tilde{Y}$ and observe that the terminal point of the path $\left(h^{\prime},\left[c^{\prime}\right]\right) .[c]$ equals the point $h^{\prime} . c(1)$.

Property (4) This statement follows from properties (1), (2) and (3): Let $\tilde{y} \in \tilde{Y}$ and let $y \in Y$ be the image of $\tilde{y}$ by the covering map. By equivariance, the homomorphism $\widetilde{H} \rightarrow H$ maps the stabilizer $\widetilde{H}_{\tilde{y}}$ into $H_{y}$. Let $h \in H_{y}$ and let $\tilde{h} \in \tilde{H}$ be an element mapping to $h$. Then there is $g \in \pi_{1} Y$ such that $g . \tilde{y}=\tilde{h}$. $\tilde{y}$. It follows that $g^{-1} \tilde{h} \in \tilde{H}_{\tilde{y}}$ and $g^{-1} \tilde{h}$ maps to $h$. This shows that $\tilde{H} \rightarrow H$ is surjective. For injectivity, if $\tilde{h}_{1}, \tilde{h}_{2} \in \tilde{H}_{\tilde{y}}$ map to $h \in H_{y}$, then $\tilde{h}_{1}^{-1} \tilde{h}_{2} \in \pi_{1} Y \cap \tilde{H}_{\tilde{y}}$. Since $\pi_{1} Y$ acts freely on $Y$, it follows that $\tilde{h}_{1}=\tilde{h}_{2}$.

Property (5) The isomorphism is a consequence of the short five lemma. Suppose that $G$ is another group acting on $\tilde{Y}$ and satisfying properties one to three; the fourth property is not needed as it is a consequence of the other three. By property (1) of $G$, there is a short exact sequence

$$
1 \rightarrow K \rightarrow G \stackrel{\varphi}{\rightarrow} H \rightarrow 1,
$$

where $K \cong \pi_{1}(Y)$. Denote by $\rho$ the covering map $\tilde{Y} \rightarrow Y$, and let $\tilde{y}_{0} \in \tilde{Y}$ be such that $\rho\left(\tilde{y}_{0}\right)=y_{0}$. For $g \in G$, let $c_{g}$ denote a path in $Y$ starting at $y_{0}$ obtained by composing $\rho$ with a path in $\tilde{Y}$ from $\tilde{y}_{0}$ to $g \cdot \tilde{y}_{0}$; here we use that $G$ acts on $\tilde{Y}$. Since $\tilde{Y}$ is simply connected, the homotopy class $\left[c_{g}\right]$ depends only on $g$; by property (2) of $G$, the pair $\left(\varphi(g),\left[c_{g}\right]\right)$ is an element of $\widetilde{H}$. It follows that there is a well-defined group homomorphism

$$
\Phi: G \rightarrow \tilde{H}, \quad g \mapsto\left(\varphi(g),\left[c_{g}\right]\right) .
$$

Observe that $\Phi(K)$ is a subset of the kernel of $\widetilde{H} \rightarrow H$ and therefore $\Phi$ satisfies the following commutative diagram:

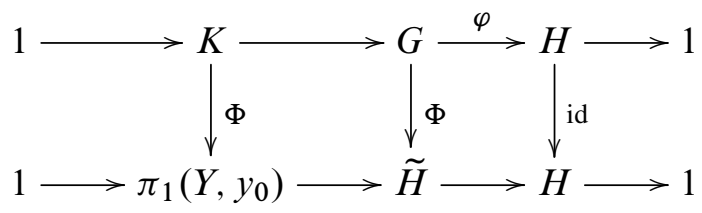


We claim that the $\Phi: K \rightarrow \pi_{1}\left(Y, y_{0}\right)$ is an isomorphism. By property (3) of $G$, the action of $K$ on $\tilde{Y}$ is the action of deck transformations of $\tilde{Y} \rightarrow Y$. Therefore for $[c] \in \pi_{1}\left(Y, y_{0}\right)$, there is $k \in K$ acting on $\tilde{Y}$ as $(1,[c])$ does. Recall that $[c]$ is identified with $(1,[c]) \in \tilde{H}$. By definition of $\Phi$, we have that $\Phi(k)=(\varphi(k),[c])=(1,[c])$ and hence $\Phi: K \rightarrow \pi_{1}\left(Y, y_{0}\right)$ is surjective. For injectivity, observe that if $k \in K$ and $\Phi(k)$ is trivial, then $k \in G_{\tilde{y}_{0}}$; then, by property (3) of $G, k$ is trivial. By the short five lemma applied to the commutative diagram above, $\Phi: G \rightarrow \widetilde{H}$ is an isomorphism.

Now we verify that the actions of $\tilde{H}$ and $G$ are identical up to composing with $\Phi$, ie, for every $g \in G$ and $\tilde{y} \in \tilde{Y}, g \cdot \tilde{y}=\Phi(g) \cdot \tilde{y}$. Suppose that $\tilde{y}=\left[c^{\prime}\right]$. Then $g \cdot \tilde{y}=\left[c_{g} * \varphi(g) \cdot c^{\prime}\right]$ since $c_{g} * \varphi(g) . c^{\prime}$ lifts to a path from $\tilde{y}_{0}$ to $g \cdot \tilde{y}$. Hence $g \cdot \tilde{y}=$ $\left[c_{g} * \varphi(g) \cdot c^{\prime}\right]=\left(\varphi(g),\left[c_{g}\right]\right) \cdot\left[c^{\prime}\right]$.

Property (6) To simplify notation, we denote by $f$ the map $X \rightarrow Y$ and the group homomorphism $G \rightarrow H$. Let $x_{0} \in X$ be such that $f\left(x_{0}\right)=y_{0}$. Since $X$ is simply connected, using the description of $\tilde{Y}$ as a set of equivalence classes of paths, for any $x \in X$ we have that $\tilde{f}(x)=[f \circ c]$ where $c$ is any path in $X$ from $x_{0}$ to $x$. We show that $\tilde{f}: X \rightarrow \tilde{Y}$ is equivariant with respect to the group homomorphism

$$
\tilde{f}: G \rightarrow \tilde{H}, \quad g \mapsto(f(g),[f \circ c]),
$$

where $c$ is a path in $X$ from $x_{0}$ to $g \cdot x_{0}$. Observe that $\tilde{f}$ is well-defined as a map since $X$ is simply connected and $f: X \rightarrow Y$ is an equivariant map. To show that $\tilde{f}$ is a homomorphism is routine. To verify equivariance, first let $x \in X$ and $g \in G$. Let $c$ be a path from $x_{0}$ to $g . x$, let $c^{\prime}$ be a path from $x_{0}$ to $g \cdot x_{0}$, and let $c^{\prime \prime}$ be a path from $x_{0}$ to $x$. Since $X$ is simply connected, we have that $\left[c^{\prime} * g . c^{\prime \prime}\right]=[c]$ and hence

$$
\begin{aligned}
\tilde{f}(g) \cdot \tilde{f}(x)=\left(f(g),\left[f \circ c^{\prime}\right]\right) \cdot\left[f \circ c^{\prime \prime}\right] & =\left[\left(f \circ c^{\prime}\right) *\left(f(g) \cdot\left(f \circ c^{\prime \prime}\right)\right)\right] \\
& =\left[f\left(c^{\prime} * g \cdot c^{\prime \prime}\right)\right]=[f(c)]=\tilde{f}(g \cdot x) .
\end{aligned}
$$

This completes the proof of the theorem.

\section{Maximal equivariant towers}

For the rest of the paper, all spaces are combinatorial complexes and all maps are combinatorial. All group actions on complexes are by combinatorial maps. A $G$-complex $X$ is proper (respectively cocompact, free) if the $G$-action is proper (respectively, cocompact, free). For a cell $\sigma$ of $X$, the pointwise $G$-stabilizer of $\sigma$ is denoted by $G_{\sigma}$ and the $G$-orbit of $\sigma$ is denoted by $G(\sigma)$. 
Definition 3.1 (Combinatorial complexes and maps [3, Chapter I, Appendix] ) A map $X \rightarrow Y$ between CW-complexes is combinatorial if its restriction to each open cell of $X$ is a homeomorphism onto an open cell of $Y$. A CW-complex $X$ is combinatorial provided that the attaching map of each open cell is combinatorial for a suitable subdivision.

\subsection{Definition of equivariant tower and basic lemmas}

Definition 3.2 (Equivariant map) Let $X$ be a $G$-complex and let $Y$ be an $H-$ complex. An equivariant map $f: X \rightarrow Y$ is a pair $\left(f, f_{\#}\right)$, where $f: X \rightarrow Y$ is a combinatorial map, $f_{\#}: G \rightarrow H$ is a group homomorphism and $f$ is equivariant with respect to $f_{\#}$, that is, $f(g . x)=f_{\#}(g) f(x)$ for every $g \in G$ and $x \in X$. As usual, if both $X$ and $Y$ are $G$-complexes and $f_{\#}$ is the identity map on $G$, then $f$ is called a $G$-map. As a convention, we use bold letters to denote equivariant maps $\boldsymbol{f}$, and in this case, we use $f$ to denote the map between complexes, and $f_{\#}$ to denote the group homomorphism. The domain of an equivariant map is the space together with the group acting on it, and the same convention applies to the codomain. The composition of equivariant maps is defined in the natural way. Equality of equivariant maps $\boldsymbol{f}=\boldsymbol{g}$ means their domains and codomains are equal, $f=g$ and $f_{\#}=g_{\#}$.

Definition 3.3 (Equivariant isomorphism) An equivariant map is an isomorphism if the map at the level of spaces is a homeomorphism and the map a the level of groups is a group isomorphism.

Definition 3.4 (Equivariant inclusions) An equivariant map $\boldsymbol{\imath}=\left(\iota, l_{\#}\right)$ is called an equivariant inclusion if $l$ and $l_{\#}$ are injective. The equivariant inclusion $\boldsymbol{l}$ is called proper if either $l$ or $l_{\#}$ is not surjective.

Definition 3.5 (Equivariant cover) Let $Y$ be an $H$-complex. A covering map $\hat{Y} \rightarrow Y$ is called an equivariant cover if $\hat{Y}$ is an $H$-regular cover. By Corollary 2.5 , if $\rho$ is an equivariant cover then there is a well defined group homomorphism $\rho_{\#}: \hat{H} \rightarrow H$ such that $\left(\rho, \rho_{\#}\right)$ is an equivariant map. When referring to an equivariant cover we will be implicitly referring to the associated equivariant map $\left(\rho, \rho_{\#}\right)$.

Definition 3.6 (Towers and $\mathcal{F}$-towers) Let $X$ be a $G$-complex and let $Y$ be an $H$-complex. An equivariant map $g: X \rightarrow Y$ is an equivariant tower if it can be expressed as an alternating composition of equivariant inclusions and equivariant covers. Specifically, $g$ is a composition

$$
X=X_{n} \hookrightarrow \hat{X}_{n} \rightarrow X_{n-1} \hookrightarrow \cdots \hookrightarrow \widehat{X}_{2} \rightarrow X_{1} \hookrightarrow \widehat{X}_{1} \rightarrow X_{0}=Y
$$


and $g_{\#}$ is a composition

$$
G=G_{n} \hookrightarrow \widehat{G}_{n} \rightarrow G_{n-1} \hookrightarrow \cdots \hookrightarrow \widehat{G}_{2} \rightarrow G_{1} \hookrightarrow \widehat{G}_{1} \rightarrow G_{0}=H,
$$

where $X_{i}$ is a $G_{i}$-complex, $\hat{X}_{i} \rightarrow X_{i-1}$ is a $G_{i-1}$-regular cover of $X_{i-1}$ inducing the $\widehat{G}_{i}$-action on $\hat{X}_{i}, X_{i}$ is a subcomplex of $\hat{X}_{i}$ invariant under the subgroup $G_{i} \leq \hat{G}_{i}$, and both $G=G_{n}$ and $H=G_{0}$. In the case that each $X_{i}$ is a full subcomplex of $\hat{X}_{i}$, and the tower $\boldsymbol{g}$ is called an $\mathcal{F}$-tower.

The length of the tower $\boldsymbol{g}$ is the smallest value of $n$ in an expression for $\boldsymbol{g}$ as above. In particular, an equivariant inclusion or cover have length at most one. By convention, the identity map has length zero.

Definition 3.7 (Equivariant tower lifting and $\mathcal{F}$-tower lifting) Let $\boldsymbol{f}$ be an equivariant map. An equivariant tower lifting of $f$ is an equivariant map $f^{\prime}$ such that there is an equivariant tower $\boldsymbol{g}$ such that $\boldsymbol{f}=\boldsymbol{g} \circ \boldsymbol{f}^{\prime}$. The lifting is trivial if $\boldsymbol{g}$ is an equivariant isomorphism, and the lifting is maximal if the only equivariant tower lifting of $\boldsymbol{f}^{\prime}$ is the trivial one. The notions of equivariant $\mathcal{F}$-tower lifting, trivial $\mathcal{F}$-tower lifting and maximal $\mathcal{F}$-tower lifting are defined analogously.

Remark 3.8 (Composition of towers) Observe that if $\boldsymbol{f}$ and $\boldsymbol{h}$ are equivariant towers, and the codomain of $\boldsymbol{f}$ equals the domain of $\boldsymbol{h}$ (this means on the space and the group), then the composition $\boldsymbol{h} \circ \boldsymbol{f}$ is an equivariant tower. The same statement holds for $\mathcal{F}$-towers.

Suppose $\boldsymbol{f}=\boldsymbol{g} \circ \boldsymbol{f}^{\prime}$ is a tower lifting of $\boldsymbol{f}$ and $\boldsymbol{f}^{\prime}=\boldsymbol{g}^{\prime} \circ \boldsymbol{f}^{\prime \prime}$ is a tower lifting of $\boldsymbol{f}^{\prime}$. Since the composition $g \circ g^{\prime}$ is a tower, $f=\left(g \circ g^{\prime}\right) \circ f^{\prime \prime}$ is a tower lifting of $\boldsymbol{f}$. In particular, if $\boldsymbol{f}^{\prime \prime}$ is a maximal tower lifting of $\boldsymbol{f}^{\prime}$ then $\boldsymbol{f}^{\prime \prime}$ is a maximal tower lifting of $\boldsymbol{f}$. The same statement holds for $\mathcal{F}$-towers liftings.

Definition 3.9 (0-surjective) A map $X \rightarrow Y$ is 0 -surjective if every 0 -cell of $Y$ is in the image of $X$.

Proposition 3.10 (Maximality $\Leftrightarrow$ surjectivity) Let $X$ be a one-connected and $G-$ cocompact complex and let $f: X \rightarrow Y$ be a $G$-map.

(1) An equivariant tower lifting $\boldsymbol{f}^{\prime}$ of $\boldsymbol{f}$ is maximal if and only if $f^{\prime}$ is surjective and $\pi_{1}$-surjective, and $f_{\#}^{\prime}$ is surjective.

(2) An equivariant $\mathcal{F}$-tower lifting $\boldsymbol{f}^{\prime}$ of $\boldsymbol{f}$ is maximal if and only if $f^{\prime}$ is 0 surjective and $\pi_{1}$-surjective, and $f_{\#}^{\prime}$ is surjective. 
Proof We sketch the proof for $\mathcal{F}$-towers and the proof of the first statement is left to the reader. The "only if" part is immediate since otherwise $f^{\prime}$ would factor through a non-trivial inclusion or the universal covering map. For the "if" part, let $\boldsymbol{f}^{\prime}=\boldsymbol{h} \circ \boldsymbol{f}^{\prime \prime}$ be an $\mathcal{F}$-tower lifting of $\boldsymbol{f}^{\prime}$. Suppose that $\boldsymbol{h}$ is an equivariant inclusion of a fullsubcomplex; since $f^{\prime}$ is 0 -surjective and $f_{\#}^{\prime}$ is surjective we have that $h$ and $h_{\#}$ are surjective and hence $\boldsymbol{h}$ is an equivariant isomorphism. Suppose that $\boldsymbol{h}$ is an equivariant cover; since $X$ is one-connected and $f^{\prime}: X \rightarrow Y^{\prime}$ is $\pi_{1}$-surjective it follows that $Y^{\prime}$ is one-connected and hence $h$ is the trivial cover and $h_{\#}$ is an isomorphism. The general case follows by induction on the length of the tower.

Definition 3.11 (Preserving 0-stabilizers) An equivariant map $f$ from the $G$-complex $X$ to the $H$-complex $Y$ is said to preserve 0 -stabilizers if for every 0 -cell $\sigma$ of $X$ the map $f_{\#}: G_{\sigma} \rightarrow H_{f(\sigma)}$ is a group isomorphism.

Definition 3.12 $(d(\boldsymbol{f}), r(\boldsymbol{f}), e(X))$ For a $G$-complex $X$, let $v(G, X)$ denote the number of $G$-orbits of 0 -cells and let $e(G, X)$ denote the number of $G$-orbits of 1 -cells. If the group is understood, we simply write $v(X)$ and $e(X)$. Observe that $v(G, X)<\infty$ and $e(G, X)<\infty$ if $X$ is $G$-cocompact. If $\boldsymbol{f}: X \rightarrow Y$ is an equivariant map from the $G$-complex $X$ to the $f_{\#}(G)$-complex $Y$, we define $d(f)=v(G, X)$ and $r(\boldsymbol{f})=v\left(f_{\#}(G), f(X)\right)$. Observe that for a $G$-map $f: X \rightarrow Y$, we have that $d(\boldsymbol{f}) \geq r(\boldsymbol{f})$ and $e(X) \geq e(f(X))$.

Lemma 3.13 Let $g: X \rightarrow Y$ be an equivariant tower such that $g_{\#}$ is an isomorphism, preserves 0 -stabilizers, and $\infty>d(\boldsymbol{g})=r(\boldsymbol{g})$. The following statements hold.

(1) The map $g$ is an equivariant inclusion and when restricted to the 0 -skeletons is an isomorphism.

(2) If $g$ is surjective, then $g$ is an equivariant isomorphism.

(3) If $\infty>e(X)=e(Y)$, then $g$ is an isomorphism when restricted to 1-skeletons and, in particular, $g$ is $\pi_{1}$-surjective.

Proof Since $g_{\#}$ is an isomorphism, assume that $X$ and $Y$ are $G$-complexes and $g_{\#}: G \rightarrow G$ is the identity map. Observe that the second statement is immediate if the first statement holds.

For the first statement, we show first that $\boldsymbol{g}$ induces a bijection between the 0 -skeletons. Since $\infty>d(\boldsymbol{g})=r(\boldsymbol{g})$, the pigeon-hole argument shows that $\boldsymbol{g}$ induces a bijection between $G$-orbits of 0 -cells. Hence it is enough to show that for any 0 -cell $x$ in the domain of $g$, the induced map between orbits $g: G(x) \rightarrow G(g(x))$ is a bijection. By equivariance, $g: G(x) \rightarrow G(g(x))$ is surjective. For injectivity, suppose there are 
$x, x^{\prime} \in X$ and $h \in G$ such that $h . x=x^{\prime}$ and $g(x)=g\left(x^{\prime}\right)$. By equivariance, $h$ is in the $G$-stabilizer of $g(x)$. Since $g_{\#}: G_{x} \rightarrow G_{g(x)}$ is the identity map, it follows that $h \in G_{x}$ and hence $x^{\prime}=x$. We have proved that $g$ induces a bijection between the 0 -skeletons. Now we show that $g$ is an inclusion of complexes. Let $\sigma$ and $\sigma^{\prime}$ be two $k$-cells of $X$ mapping to the same $k$-cell of $Y$. Since $g$ is bijective on 0 -cells, the cells $\sigma$ and $\sigma^{\prime}$ have a common 0 -cell in their closure. Since $g$ is a tower, it is a locally injective map. Therefore $\sigma$ and $\sigma^{\prime}$ are the same $k$-cell.

The third statement is proved as follows. The first statement of the lemma implies that $\boldsymbol{g}$ induces an equivariant inclusion between the 1 -skeletons and an isomorphism between 0 -skeletons. It remains to prove that the induced map between $1-$ skeletons is surjective. Since $\infty>e(X)=e(Y)$, the pigeon-hole argument shows that $\boldsymbol{g}$ induces a bijection between $G$-orbits of 1 -cells. For any 1 -cell $\sigma$, equivariance implies that the induced map between orbits $g: G(\sigma) \rightarrow G(g \sigma)$ is surjective. Therefore $g$ is surjective on 1-cells, and hence the induced map between the 1 -skeletons is an isomorphism.

\subsection{Existence of maximal $\mathcal{F}$-tower liftings}

Theorem 3.14 (Maximal equivariant $\mathcal{F}$-towers) Let $f: X \rightarrow Y$ be a $G$-map. If $X$ is one-connected and $G$-cocompact and $Y$ is locally finite, then $f$ has a maximal equivariant $\mathcal{F}$-tower lifting.

Before the proof of the theorem we need a definition and a remark.

Definition 3.15 (Span) The span of a subcomplex $K \subset X$, denoted by $\operatorname{Span}_{X}(K)$, is the smallest full subcomplex of $X$ containing $K$.

Remark 3.16 If $Y$ is a $G$-complex and $K \subset Y$ is a $G$-subcomplex, then $\operatorname{Span}_{X}(K)$ is a $G$-subcomplex of $Y$. If, in addition, $Y$ is locally finite and $K$ is $G$-cocompact then $\operatorname{Span}_{X}(K)$ is $G$-cocompact and in particular $e\left(\operatorname{Span}_{X}(K)\right)$ is finite.

Proof of Theorem 3.14 Let $\boldsymbol{f}_{0}$ denote the $G$-map $\boldsymbol{f}$. Let $Y_{1}$ be the $G$-subcomplex Span $(f(X))$ of $Y$, let $f_{1}: X \rightarrow Y_{1}$ by the $G$-map defined as $f_{1}(x)=f(x)$ for $x \in X$ and let $g_{1}: Y_{1} \rightarrow Y$ be the $G$-equivariant inclusion. Since $X$ is $G$-cocompact, $\infty>d\left(f_{1}\right)-r\left(f_{1}\right)$. Since $Y$ is locally finite and $X$ is $G$-cocompact, $Y_{1}$ is locally finite and $\infty>e\left(Y_{1}\right)$.

For $n \geq 1$, suppose that for we have defined an $\mathcal{F}$-tower lifting $f_{n}: X \rightarrow Y_{n}$ of $f_{n-1}$ and an $\mathcal{F}$-tower $\boldsymbol{g}_{n}: Y_{n} \rightarrow Y_{n-1}$ such that $\boldsymbol{g}_{n} \circ \boldsymbol{f}_{n}=\boldsymbol{f}_{n-1}$ and $Y_{n}$ is locally finite and $\infty>d\left(f_{n}\right)-r\left(f_{n}\right)$ and $\infty>e\left(Y_{n}\right)$. Let $\rho_{n}: \tilde{Y}_{n} \rightarrow Y_{n}$ be the universal covering 
map and let $\tilde{f}_{n}: X \rightarrow \tilde{Y}_{n}$ be the equivariant lifting of $\boldsymbol{f}_{n}$ to the universal cover $\tilde{Y}_{n}$. By Theorem 2.2, $\tilde{f}_{n}$ is naturally a $G$-map. Let $Y_{n+1}$ be the $G$-subcomplex $\operatorname{Span}\left(\tilde{f}_{n}(X)\right)$ of $\tilde{Y}_{n}$ and let $f_{n+1}: X \rightarrow Y_{n+1}$ the $G$-map defined by $f_{n+1}(x)=\tilde{f}_{n}(x)$ for each $x \in X$. Let $g_{n+1}: Y_{n+1} \rightarrow Y_{n}$ be the restriction of $\rho_{n}$ to $Y_{n+1}$. By construction, $f_{n+1}: X \rightarrow Y_{n+1}$ is 0 -surjective. Since $\tilde{Y}_{n}$ is locally finite and $X$ is $G$-cocompact, $Y_{n+1}$ is also locally finite and $\infty>e\left(Y_{n+1}\right)$. The construction is illustrated in Figure 1.

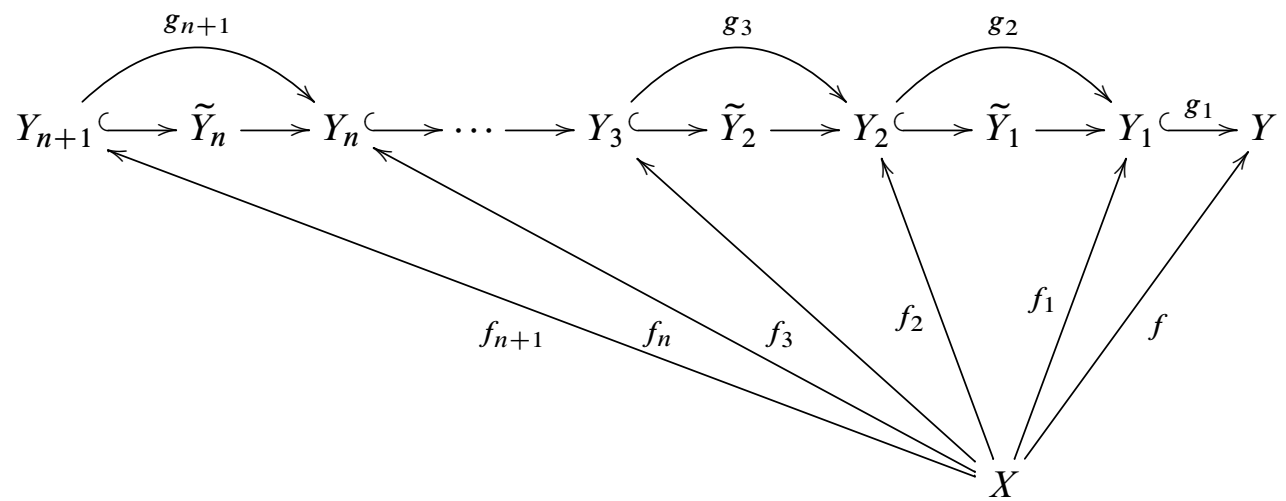

Figure 1: The tower construction in the proof of Theorem 3.14

Consider the well-ordered set consisting of pairs of positive integers $\mathbb{N} \times \mathbb{N}$ with the dictionary order $\leq_{\text {dic }}$. For each $f_{n}$ we assign an element $\left|f_{n}\right|$ of $\mathbb{N} \times \mathbb{N}$ defined as

$$
\left|f_{n}\right|=\left(d(f)-r\left(f_{n}\right), e\left(Y_{n}\right)\right) .
$$

Lemma 3.17 (Decreasing complexity) The following statements hold.

(1) If $Y_{n}$ is simply connected, then $f_{n}: X \rightarrow Y_{n}$ is a maximal $\mathcal{F}$-tower lifting of $f: X \rightarrow Y$.

(2) If $\left|\boldsymbol{f}_{n+1}\right|={ }_{\mathrm{dic}}\left|\boldsymbol{f}_{n}\right|$, then $Y_{n}$ is simply connected.

(3) For every $n,\left|f_{n+1}\right| \leq_{\text {dic }}\left|f_{n}\right|$.

Proof If $Y_{n}$ is simply connected then $f_{n}$ is $\pi_{1}$-surjective. Since $f_{n}$ is also $0-$ surjective, Proposition 3.10 implies that $f_{n}$ is a maximal $\mathcal{F}$-tower lifting of $\boldsymbol{f}$. This proves the first statement.

For the second statement, suppose $\left|\boldsymbol{f}_{n+1}\right|=_{\text {dic }}\left|\boldsymbol{f}_{n}\right|$. We will show that $g_{n+1}: Y_{n+1} \rightarrow$ $Y_{n}$ is $\pi_{1}$-surjective using Lemma 3.13(3); then the proof concludes by observing that $g_{n+1}$ factors through the simply connected space $\tilde{Y}_{n}$ and hence $Y_{n}$ is simply 
connected. It remains to verify that $\boldsymbol{g}_{n+1}$ satisfies the hypotheses of Lemma 3.13(3). By construction, $g_{n+1}: Y_{n+1} \rightarrow Y_{n}$ is a $G-$ map and a tower; the assumption implies that $\infty>d\left(\boldsymbol{g}_{n+1}\right)=r\left(\boldsymbol{f}_{n+1}\right)=r\left(\boldsymbol{f}_{n}\right)=r\left(\boldsymbol{g}_{n+1}\right)$ and $\infty>e\left(Y_{n+1}\right)=e\left(Y_{n}\right)$; an induction argument together with Theorem 2.2(4) shows that $\boldsymbol{g}_{n+1}$ preserves 0 stabilizers for $n \geq 1$.

The third statement is proved as follows. First observe that

$$
\infty>d(\boldsymbol{f})=d\left(\boldsymbol{f}_{n+1}\right) \geq r\left(\boldsymbol{f}_{n+1}\right)=d\left(\boldsymbol{g}_{n+1}\right) \geq r\left(\boldsymbol{g}_{n+1}\right) \geq r\left(\boldsymbol{f}_{n}\right) \geq 0,
$$

and hence $d(\boldsymbol{f})-r\left(f_{n}\right) \geq d(\boldsymbol{f})-r\left(f_{n+1}\right) \geq 0$. If $r\left(f_{n}\right)=r\left(f_{n+1}\right)$, then $d\left(g_{n+1}\right)=$ $r\left(g_{n+1}\right)$. Then Lemma 3.13(1) implies that $\boldsymbol{g}_{n+1}: Y_{n+1} \rightarrow Y_{n}$ is a $G$-equivariant inclusion and therefore $e\left(Y_{n}\right) \geq e\left(Y_{n+1}\right)$. It follows that $\left|f_{n+1}\right| \leq_{\text {dic }}\left|f_{n}\right|$.

To conclude the proof of Theorem 3.14 observe that if for every $n$ the complex $Y_{n}$ is not simply connected, then there is an infinite strictly decreasing sequence of elements of $\left(\mathbb{N} \times \mathbb{N}, \leq_{\text {dic }}\right)$. Since this is impossible, there is $n$ such that $Y_{n}$ is simply connected, and then $f_{n}$ is a maximal $\mathcal{F}$-tower lifting of $\boldsymbol{f}$.

\subsection{Existence of maximal towers liftings}

Theorem 3.18 (Maximal equivariant towers) Let $f: X \rightarrow Y$ be a $G$-map. If $X$ is one-connected and $G$-cocompact, then $f$ has a maximal equivariant tower lifting.

Sketch of the proof The proof of Theorem 3.18 is the same as the proof of Theorem 3.14 with some simplifications. Define inductively the sequence of tower liftings $f_{n+1}: X \rightarrow Y_{n+1}$ where $Y_{n}$ is defined as the $G$-subcomplex $\tilde{f}_{n}(X)$ of $\tilde{Y}_{n}$ and $Y_{1}=f(X)$. Since $X$ is $G$-cocompact and $f_{n}$ is surjective, $Y_{n}$ is $G$-cocompact and hence $d\left(f_{n}\right)-r\left(f_{n}\right)<\infty$. Then, the same argument shows that

$$
d\left(f_{n}\right)-r\left(f_{n}\right) \geq d\left(f_{n+1}\right)-r\left(f_{n+1}\right) .
$$

One verifies that if $Y_{n}$ is not simply connected then the inequality above is strict. Specifically, if $r\left(f_{n}\right)=r\left(f_{n+1}\right)$ then $g_{n+1}$ is a surjective $G$-equivariant tower preserving 0-stabilizers and such that $d\left(\boldsymbol{g}_{n+1}\right)=r\left(\boldsymbol{g}_{n+1}\right)$; then Lemma 3.13(2) implies that $g_{n+1}$ is an isomorphism factoring through $\tilde{Y}_{n}$ and hence $Y_{n}$ is simply connected. If each $Y_{n}$ is not simply connected then $d\left(f_{n}\right)-r\left(f_{n}\right)$ defines a strictly decreasing infinite sequence of natural numbers which is impossible. Therefore some $Y_{n}$ is simply connected and the corresponding $f_{n}$ is the desired maximal tower lifting of $f$. 


\section{Immersed cocompact cores}

Theorem 4.1 If $Y$ is a one-connected, proper and locally finite $H$-complex and $G \leq H$ is finitely presented, then there is a one-connected cocompact $G$-complex $X$ and a $G$-equivariant $\mathcal{F}$-tower $X \rightarrow Y$.

The proof of the theorem requires two lemmas.

Lemma 4.2 Let $\mathcal{G}$ be a finite graph of groups such that vertex groups are finitely generated and edge groups are finite. If $\varphi: \pi_{1}(\mathcal{G}) \rightarrow G$ is a surjective group homomorphism into a finitely presented group, then $\operatorname{Kernel}(\varphi)$ is normally finitely generated.

Proof The hypotheses on $\mathcal{G}$ imply that $\pi_{1}(\mathcal{G})$ is finitely generated. Therefore there is a surjective homomorphism $\psi: F \rightarrow \pi_{1}(\mathcal{G})$, where $F$ is a finite-rank free group. Since $G$ is finitely presented and $F$ has finite rank, the kernel of $\varphi \circ \psi$ is normally finitely generated, say Kernel $(\varphi \circ \psi)=\left\langle\left\langle r_{1}, \ldots, r_{m}\right\rangle\right\rangle$. Since $\operatorname{Kernel}(\varphi)=\psi(\operatorname{Kernel}(\varphi \circ \psi))$, we have that $\operatorname{Kernel}(\varphi)=\left\langle\left\langle\psi\left(r_{1}\right), \ldots, \psi\left(r_{m}\right)\right\rangle\right\rangle$.

Recall that a group is slender if all its subgroups are finitely generated, and a $G$-complex is almost proper if $G$ acts properly on the complement of the 0 -skeleton.

Lemma 4.3 (One-connected complex for finitely presented subgroup) Let $Y$ be a one-connected, almost proper $H$-complex such that $H$-stabilizers of cells are slender. Suppose that $G \leq H$ is finitely presented. Then there exists a cocompact and oneconnected $G-2$-complex $X$, and an equivariant map $f: X \rightarrow Y$ such that $f_{\#}$ is the inclusion $G \hookrightarrow H$ and $f_{\#}$ is injective when restricted to 0 -cell stabilizers.

Proof First we construct the 1-skeleton $X^{(1)}$ of $X$ as a $G$-equivariant cocompact subcomplex of $Y$. Let $\left\{g_{i}\right\}_{i=1}^{m}$ be a finite generating set for $G$ and let $y_{0}$ be a 0 -cell of $Y$. Since $Y$ is connected, for each $1 \leq i \leq m$ there is a combinatorial path $\gamma_{i}$ from $y_{0}$ to $g_{i} y_{0}$. Let $D$ be the finite subcomplex $D=\gamma_{1} \cup \cdots \cup \gamma_{m}$ of $Y$ and let $X^{(1)}$ be the union of all $G$-translates of $D$ in $Y$. Then $X^{(1)}$ is a $G$-equivariant 1-dimensional cocompact connected subcomplex of $Y$. Connectedness follows from the assumption that $\left\{g_{i}\right\}_{i=1}^{m}$ generates $G$, and cocompactness from $D$ being a finite subcomplex.

Next we show that $\pi_{1} X^{(1)}$ is a normally finitely generated group. Invoking Theorem 2.2, consider the group $\widetilde{G}$ acting on the universal cover $\mathcal{T}$ of $X^{(1)}$ such that $\mathcal{T} \rightarrow X^{(1)}$ is equivariant with respect to $\widetilde{G} \rightarrow G$ and $\pi_{1} X^{(1)}$ is isomorphic to the kernel of $\widetilde{G} \rightarrow G$. By considering the barycentric subdivision $\mathcal{T}^{\prime}$ of $\mathcal{T}$, we have a cocompact action without inversions of $\widetilde{G}$ on the tree $\mathcal{T}^{\prime}$; here cocompactness follows from $X^{(1)}$ 
being $G$-cocompact. By Theorem 2.2(4) the group homomorphism $g_{\#}: \widetilde{G}_{x} \rightarrow G_{g(x)}$ is injective for every $x \in \mathcal{T}$. Therefore 1 -cell $\widetilde{G}$-stabilizers of $\mathcal{T}^{\prime}$ are finite, since $Y$ is an almost proper $G$-complex. Moreover, 0 -cell $\widetilde{G}$-stabilizers of $\mathcal{T}^{\prime}$ are isomorphic to either a subgroup of the $G$-stabilizer of a 0 -cell of $Y$ and hence finitely generated by the slender hypothesis, or to a subgroup of the setwise $\widetilde{G}$-stabilizer of a 1 -cell of $Y$ and hence finite since $Y$ is almost proper. Then the theory of Bass and Serre on actions on trees [22] implies that $\widetilde{G}$ is isomorphic to the fundamental group of a finite graph of groups $\mathcal{G}$ with finite edge groups and finitely generated vertex groups. By Lemma 4.2 , the kernel of $\widetilde{G} \rightarrow G$ is normally finitely generated.

To conclude the proof, we paste finitely many $G$-orbits of 2-cells to $X^{(1)}$ to obtain a one-connected complex. Choose a $0-$ cell $\tilde{x}_{0}$ of $\mathcal{T}$ as a basepoint. Since $\pi_{1} X^{(1)}$ is normally finitely generated, there is a finite collection $\left\{r_{i}\right\}_{i=1}^{q}$ of based loops in $X^{(1)}$ such that $\pi_{1} X^{(1)}=\left\langle\left\langle r_{1}, \ldots, r_{q}\right\rangle\right\rangle$. Since $Y$ is simply connected and $X^{(1)}$ is a subcomplex of $Y$, for each $r_{i}$ there is a disk-diagram $D_{i} \rightarrow Y$ with boundary path $r_{i}$. Let $X$ be the complex obtained by attaching a copy of $D_{i}$ to $X^{(1)}$ along the closed path $g . r_{i}$ for each $g \in G$ and each $1 \leq i \leq q$. Observe that $X$ is connected and simply connected, and the $G$-action on $X^{(1)}$ naturally extends to a cocompact $G$-action on $X$. The equivariant inclusion $X^{(1)} \hookrightarrow Y$ extends to a map $X \rightarrow Y$ equivariant with respect to $G \hookrightarrow H$.

The proof of the Theorem 4.1 is an application of Lemma 4.3 together with Theorem 3.14.

Proof of Theorem 4.1 Observe that the hypotheses of Lemma 4.3 are satisfied since $Y$ being proper implies that stabilizers of 0 -cells are finite and proper implies almost proper. Therefore there is a one-connected cocompact $G$-complex $X_{0}$ and a $G$-map $\boldsymbol{f}: X_{0} \rightarrow Y$. By Theorem 3.14, there is a maximal $\mathcal{F}$-tower lifting $\boldsymbol{f}=\boldsymbol{g} \circ \boldsymbol{f}^{\prime}$ of $\boldsymbol{f}$, where g: $X \rightarrow Y$. By Proposition 3.10(2), $f^{\prime}$ is 0 -surjective and $\pi_{1}$-surjective, and $f_{\#}^{\prime}$ is surjective. It follows that $X$ is one-connected and $f_{\#}^{\prime}$ is an isomorphism; in particular, $\boldsymbol{f}^{\prime}$ is a $G$-map. Since $Y$ is locally finite and $\boldsymbol{g}$ is a tower, it follows that $X$ is locally finite. Since $X_{0}$ is $G$-cocompact and $f^{\prime}: X_{0} \rightarrow X$ is 0 -surjective, we have that $X$ is $G$-cocompact.

Analogously to the argument in the proof of Theorem 4.1, one obtains the following result by combining Lemma 4.3 and Theorem 3.18. In this result, the complex $Y$ is not necessarily locally finite but the conclusion is weaker.

Theorem 4.4 Let $Y$ be a one-connected and almost proper $H$-complex such that $H$-stabilizers of cells are slender. If $G \leq H$ is finitely presented, then there is a one-connected cocompact $G$-complex $X$ and a $G$-equivariant tower $X \rightarrow Y$. 
A version of Theorem 4.4 appears in Martínez-Pedroza and Wise [19, Lemma 6.4] where is shown that a $G$-map with locally finite target always factors as the composition of a surjective and $\pi_{1}$-surjective $G$-map followed by a $G$-equivariant immersion.

\section{Applications}

\subsection{Subgroups of diagrammatically reducible groups}

Definition 5.1 (Near-immersion) A map $X \rightarrow Y$ is a near immersion if it is locally injective in the complement of the 0 -skeleton of $X$.

Definition 5.2 (Diagrammatically reducible complex, [7]) A 2-complex $X$ is diagrammatically reducible if there are no near-immersions $C \rightarrow X$, where $C$ is a cell structure for the 2-dimensional sphere.

First we recall some properties of diagrammatically reducible complexes in the proposition below.

Proposition 5.3 (1) Diagrammatically reducible complexes are aspherical.

(2) Covers and subcomplexes of diagrammatically reducible complexes are diagrammatically reducible.

(3) The barycentric subdivision of a diagrammatically reducible complex is diagrammatically reducible.

Proof The first statement is a result of Gersten [7, Remark 3.2], the second statement is trivial since the composition of a near-immersion and an immersion is a near-immersion and the third statement is due to Howie [7, Remark 6.10].

Definition 5.4 (Free 1-cells and collapsing) Let $X$ be a 2-complex. A 1-cell $e$ of a subcomplex $Z \leq X$ is free if it belongs to the boundary of a 2-cell $f$ of $Z$, and $e$ does not belong to the boundary of a $2-$ cell $\neq f$ in $Z$. In this case, collapsing $Z$ along $e$ means to remove the interior of $e$ and the interior of $f$.

The following characterization of a diagrammatically reducible complex is a result of Jon Corson.

Theorem 5.5 (Characterization, [6, Theorem 2.1]) A one-connected 2-complex is diagrammatically reducible if and only if every finite subcomplex is 1-dimensional or contains a free $1-$ cell. 
Remark 5.6 (Equivariant collapsing and inversions) Recall that a group action on a complex has no inversions if whenever a cell is fixed setwise by a group element then it is fixed pointwise by the group element. Let $Z$ be a $G$-complex without inversions and suppose that $e$ is a free $1-$ cell of $Z$ that belongs to the boundary of the 2-cell $f$. Observe that for every $g \in G$ the $1-$ cell $g . e$ is free in $Z$. Since $G$ acts without inversions, for every $g \in G$, the 2 -cell $g$. $f$ contains only one 1 -cell in the $G$-orbit of $e$, namely g.e. Therefore we can simultaneously collapse $Z$ along $g$.e for every $g \in G$ obtaining a $G$-equivariant subcomplex $Z^{\prime}$ of $X$.

Corson also proves that if $F$ is a finite group acting on a one-connected diagrammatically reducible 2-complex $X$ then the fixed point set $X^{F}$ of $F$ is non-empty [6, Theorem 4.1]. The following proposition shows that $X^{F}$ is also contractible.

Proposition 5.7 (Contractible fixed point sets) Let $X$ be a one-connected diagrammatically reducible 2-complex. If $F$ is a finite group acting on $X$ without inversions, then the fixed point set $X^{F}$ of $F$ is a non-empty contractible subcomplex.

Proof Since $F$ acts without inversions, $X^{F}$ is a subcomplex of $X$. By Proposition 5.3(1), it is enough to show that $X^{F}$ is one-connected. First we verify that $X^{F}$ is connected. Take two vertices $x_{0}$ and $x_{1}$ of $X^{F}$. Since $X$ is connected, there is an edge-path $\alpha$ in $X$ between $x_{0}$ and $x_{1}$. Let $Y_{0}$ be the subcomplex of $X$ defined as the union of all the images of $\alpha$ under the action of $F$. Observe that $Y_{0}$ is a connected finite 1-dimensional subcomplex of $X$ invariant under the $F$-action. Construct a finite one-connected $F$-complex $Y$ as follows. Fix a basepoint of $Y_{0}$ and let $\gamma_{1}, \ldots, \gamma_{n}$ be a collection of closed paths in $Y_{0}$ that generate $\pi_{1}\left(Y_{0}\right)$. For each $\gamma_{i}$, there is a disk diagram $D_{i} \rightarrow X$ with boundary path $\gamma_{i} \rightarrow X$. Let $Y$ be the 2-complex obtained by attaching a copy of $D_{i}$ to $Y_{0}$ along $k \cdot \gamma_{i}$ for each $k \in F$. Then $Y$ is a finite one-connected 2-complex, the $F$-action on $Y_{0}$ extends to an action on $Y$, and there is a natural $F$-equivariant map $f: Y \rightarrow X$. By Theorem 3.18, there is a maximal equivariant tower lifting

$$
Y \stackrel{f^{\prime}}{\rightarrow} Z \stackrel{g}{\rightarrow} X
$$

of $f$. Since $g$ is an immersion, $Z$ is diagrammatically reducible. Since $F$ acts without inversions on $X$, it also acts without inversions on $Z$. By Theorem 5.5, it follows that if $Z$ contains $2-$ cells, then it has a free 1 -cell $e$. After a finite number of $F$-equivariant collapses of $Z$ one obtains a 1 -dimensional one-connected $F$-complex equivariantly immersed into $X$; see Remark 5.6. Without loss of generality, we can assume that $Z$ is 1-dimensional. Then $Z$ is a tree and therefore $F$ fixes pointwise an edge-path in $Z$ between $x_{0}$ and $x_{1}$. By equivariance of the map $Y \rightarrow X, F$ fixes pointwise a path between $x_{0}$ and $x_{1}$. 
Now we verify that $X^{F}$ is simply connected. Since $X$ is simply connected, consider an essential embedded closed path $\gamma$ in $X^{F}$ with minimal area in $X$. Then there is a disk-diagram $D \rightarrow X$ with boundary $\gamma$ of minimal area. Let $g \in F$ with $g \neq 1$ and observe that the spherical diagram $D \cup g D \rightarrow X$ is a near-immersion. Since this is impossible, $X^{F}$ is simply connected.

Remark 5.8 (Inversions and connected fixed point sets) During the review process of the article, the referee observed that in the proof of Proposition 5.7 one can prove that $X^{F}$ is connected without assuming that $F$ acts without inversions. The sketch of the argument is as follows. Suppose that $X^{F}$ is not connected and choose $x_{0}$ and $x_{1}$ at minimal distance in different connected components of $X^{F}$. Consider a path $\gamma$ in $X$ between $x_{0}$ and $x_{1}$. Since $x_{0}$ and $x_{1}$ are in different connected components, the path $\gamma$ is not fixed by $F$ and hence there is a non-trivial element $g \in F$ that does not fix $\gamma$ pointwise. For given $\gamma$ and $g \in F$, there is a disk diagram $D \rightarrow X$ with boundary path $\gamma^{-1} g(\gamma)$. Among all these possible choices of $\gamma, g$ and $D$, choose the ones that minimize $\operatorname{Area}(D)$. It follows that the diagram $D$ contains $2-$ cells and no cut-points. Let $n$ be the order of the element $g$. Then one can glue together $n$ copies of $D$, by identifying $g(\gamma)$ in the $i^{\text {th }}$ copy of $D$ with $\gamma$ in the $(i+1)^{\text {st }}$ copy of $D$, producing a sphere $S$. The map $S \rightarrow X$, which maps the $i^{\text {th }}$ copy of $D$ in $S$ to $g^{i}(D)$ in $X$ is a near-immersion by our minimality choices. This contradicts the fact that $X$ is diagrammatically reducible.

Definition 5.9 Let $G$ be a group. A proper $G$-complex $X$ is a model for $\underline{E} G$ if for every finite subgroup of $F \leq G$, the fixed point set $X^{F}$ is contractible.

Theorem 5.10 (Diagrammatically reduced groups) Let $Y$ be a diagrammatically reducible proper $H-2-$ complex. If $G \leq H$ is finitely presented then $G$ admits a diagrammatically reduced 2-dimensional cocompact model for $\underline{E} H$.

Proof By passing to a subdivision of $Y$ we can assume that $G$ acts without inversions while still assuming that $Y$ is diagrammatically reducible; see Proposition 5.3(3). By Theorem 4.4, there is an equivariant immersion $X \rightarrow Y$, where $X$ is a one-connected cocompact $G-2$-complex. Since $Y$ is diagrammatically reducible, Proposition 5.3(2) implies that $X$ is diagrammatically reducible as well. Since the $H$-action on $Y$ is proper and without inversions, the same properties hold for the $G$-action on $X$. Let $K$ be a finite subgroup of $G$. Then Proposition 5.7 implies that the fixed point set $X^{F}$ is contractible. 


\subsection{Subgroups of relatively hyperbolic groups acting on negatively curved 2-complexes}

Definition 5.11 (Fine graphs and fine complexes, Bowditch [1]) A 1-complex is fine if each 1-cell is contained in only finitely many circuits of length $n$ for each $n$. Equivalently, the number of embedded paths of length $n$ between any pair of (distinct) $0-$ cells is finite. A complex is fine if its 1 -skeleton is fine.

Definition 5.12 (Relatively hyperbolic groups [1]) A group $G$ is hyperbolic relative to a finite collection of subgroups $\mathcal{P}$ if $G$ acts cocompactly, almost properly on a connected, fine, $\delta$-hyperbolic 1 -complex, and $\mathcal{P}$ is a set of representatives of distinct conjugacy classes of vertex stabilizers such that each infinite stabilizer is represented.

Proposition 5.13 (2-dimensional relative hyperbolicity) Let $X$ be a one-connected, negatively curved, fine, cocompact and almost proper $G-2$-complex. Then $G$ is a hyperbolic group relative to a (hence any) collection of representatives of conjugacy classes of 0 -cell stabilizers.

Proof Since $X$ is negatively curved and one-connected, it is a $\delta$-hyperbolic space. Indeed, it is well known that a $C A T(\kappa)$-space with $\kappa<0$ is $\delta$-hyperbolic, and in the conformal case $X$ satisfies a linear isoperimetric inequality and hence the combinatorial metric on its one skeleton is a $\delta$-hyperbolic space [3;8]. Since $X$ is $G$-cocompact there are finitely many types of 2 -cells and hence the 1 -skeleton $X^{(1)}$ is quasi-isometric to $X$; in particular $X^{(1)}$ is a $\delta^{\prime}$-hyperbolic space. It follows that $X^{(1)}$ is endowed with a $G$-action satisfying the conditions of Definition 5.12.

Proposition 5.14 Let $Y$ be a diagrammatically reducible one-connected cocompact almost proper $H-2-$ complex with a fine one-skeleton. If $X$ is one-connected and there is an immersion $X \rightarrow Y$, then $X$ has a fine one-skeleton.

Proof We use the following characterization of simplicial fine graphs due to Brian Bowditch [1, Proposition 2.1]. By simplicial we mean no double edges and no single edge loops. A simplicial graph $K$ is fine if and only if for each vertex $x \in K$, the set $V(x)$ of vertices adjacent to $x$ has the following property: every subset of $V(x)$ that is bounded in $K \backslash\{x\}$ with respect to the combinatorial metric is finite.

Without loss of generality, assume that the boundary path of every 2-cell of $Y$ is an embedded path, and that $X$ and $Y$ have simplicial one-skeleton. Indeed, by considering the barycentric subdivisions of $X$ and $Y$, we can assume the boundary paths of 2cells are embedded and one-skeletons are simplicial. Proving the proposition for the 
barycentric subdivisions is sufficient since the one-skeleton of an almost proper $\mathrm{H}-2$ complex is fine if and only if the one-skeleton of its barycentric subdivision is fine; this follows directly from [1, Lemma 2.3] or [18, Lemma 2.9]. Moreover, a complex is diagrammatically reducible if and only if its barycentric subdivision is diagrammatically reducible; see Proposition 5.3(3).

Since $Y$ admits a cocompact $H$-action and its one skeleton is fine, for every $n \in \mathbb{N}$ there are finitely many cycles of length $n$ up to the $H$-action. Therefore, since $Y$ is simply connected, there is a well defined Dehn function $\Delta: \mathbb{N} \rightarrow \mathbb{N}$, that is, $\Delta(n)$ is an upper bound for the area of minimal area disk diagrams with given boundary path of length at most $n$.

Let $f: X \rightarrow Y$ be an immersion. Let $x_{0} \in X$ be 0 -cell of $X$ and let $A$ be a subset of 0 -cells adjacent to $X$. Denote by $\operatorname{diam}(A)$ the diameter of $A$ in $X \backslash\left\{x_{0}\right\}$ in the combinatorial metric, and analogously let $\operatorname{diam}(f(A))$ denote the diameter of $f(A)$ in $Y \backslash\left\{f\left(x_{0}\right)\right\}$ in the combinatorial metric. The claim is that if $\operatorname{diam}(A)$ is finite then

$$
\operatorname{diam}(f(A)) \leq C \cdot \Delta(\operatorname{diam}(A)+2),
$$

where $C$ is an upper bound for the boundary length of a 2-cell of $Y$; here $C$ is finite since $Y$ admits a cocompact action. Assuming the claim, we conclude using Bowditch's characterization of fineness as follows. If $\operatorname{diam}(A)$ is finite, then the claim implies that $\operatorname{diam}(f(A))$ is finite; then $Y$ being fine implies that $f(A)$ is a finite set; since $f$ is an immersion and one-skeletons are simplicial, the induced map $f: A \rightarrow f(A)$ is a bijection and hence $A$ is finite set.

Suppose that $\operatorname{diam}(A)=m<\infty$ in $X \backslash\left\{x_{0}\right\}$. Let $a, b \in A$ and suppose that $a \neq b$. Then there is a combinatorial path $\gamma$ in $X \backslash\left\{x_{0}\right\}$ from $a$ to $b$ of length at most $m$. If the path $f \circ \gamma$ in $Y$ does not contain the 0 -cell $f\left(x_{0}\right)$ then the combinatorial distance between $f(a)$ and $f(b)$ is bounded by $m$; however this assumption on $f \circ \gamma$ might not hold. A general argument is as follows. Consider the closed path $\gamma^{\prime} \rightarrow X$ defined as the concatenation $\gamma^{\prime}=e_{1} * \gamma * e_{2}$, where $e_{1}$ is an 1-cell from $x_{0}$ to $a$, and $e_{2}$ is a 1 -cell from $b$ to $x_{0}$. Since $X$ is simply connected, there is a near-immersion of a disk-diagram $D \rightarrow X$ with boundary path $\gamma^{\prime}$. Observe that there is only one 0 -cell in $\partial D$ mapping to $x_{0} \in X$; by abuse of notation, let $x_{0}$ denote this 0 -cell of $D$.

The main observation is that $D \backslash\left\{x_{0}\right\}$ is connected. Indeed, if $D \backslash\left\{x_{0}\right\}$ is not connected, then the boundary path $\partial D \rightarrow X$ passes through $x_{0}$ more than once. Since $e_{1} \rightarrow X$ and $e_{2} \rightarrow X$ are 1-cells with only one endpoint equal $x_{0}$, and the image of $\gamma \rightarrow X$ does not contain $x_{0}$, it follows that $\partial D=e_{1} * \gamma * e_{2}$ passes through $x_{0}$ only once, and hence $D \backslash\left\{x_{0}\right\}$ is connected. 
The fact that $D \backslash\left\{x_{0}\right\}$ is connected implies that there is an embedded path $\eta \rightarrow D$ between the two 0 -cells adjacent to $x_{0}$ in $\partial D$. This path $\eta \rightarrow D$ factors through $D \backslash\left\{x_{0}\right\}$ and goes around the 2-cells of $D$ adjacent to $x_{0}$; see Figure 2. Observe that

$$
\eta \rightarrow D \rightarrow X \stackrel{f}{\rightarrow} Y
$$

is a path between $f(a)$ and $f(b)$, and the combinatorial length of $\eta$ is bounded by $C \cdot \operatorname{Area}(D)$ where $C$ is the upper bound for the boundary length of a 2-cell of $Y$.

Now observe that the path

$$
\eta \rightarrow D \rightarrow X \stackrel{f}{\rightarrow} Y
$$

does not intersect $f\left(x_{0}\right)$. Indeed, since boundary paths of 2-cells of $Y$ (and hence of $D$ ) are embedded, if $\eta \rightarrow Y$ intersects $f\left(x_{0}\right)$ then there is a 2-cell $R$ of $D$ whose boundary path contains $x_{0} \in \partial D$ and another 0 -cell $x \in \eta$ both mapping to $f\left(x_{0}\right)$, this would imply that $\partial R \rightarrow Y$ is not an embedded path, which is impossible by our initial assumption.

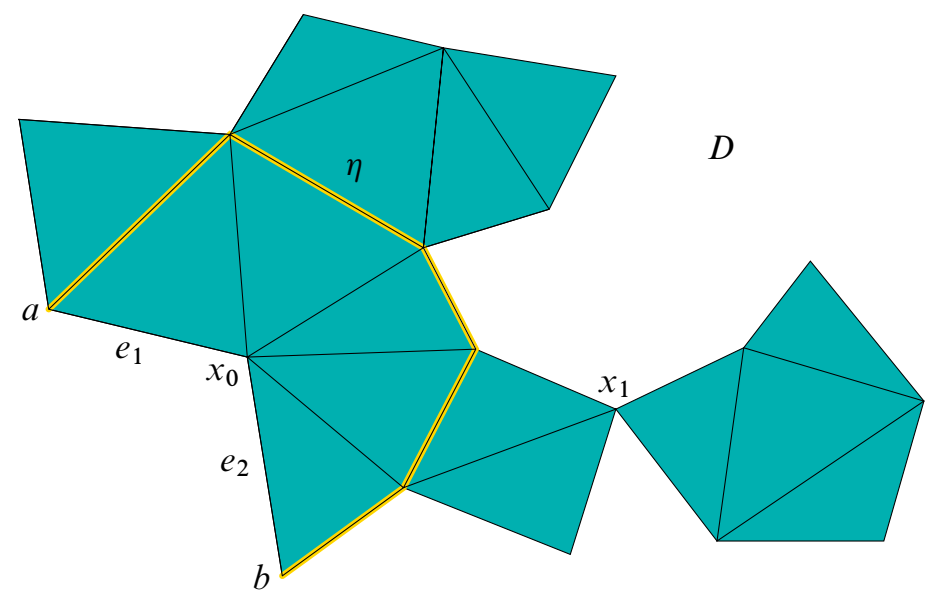

Figure 2: In the disk diagram $D$, the space $D \backslash\left\{x_{1}\right\}$ is not connected. If $D$ is a disk diagram, $x_{0}$ is a 0 -cell on $\partial D$ adjacent to the 0 -cells $a, b \in \partial D$ with $a \neq b$, and $D \backslash\left\{x_{0}\right\}$ is connected, then there is an edge path $\eta \rightarrow D$ between $a, b$ that factors through $D \backslash\left\{x_{0}\right\}$ and goes around the 2-cells of $D$ adjacent to $x_{0}$. The length of $\eta$ is bounded by $\operatorname{Area}(D) \cdot C_{1}$, where $C_{1}$ is an upper bound for the boundary length of 2-cells of $D$.

Since $Y$ is diagrammatically reducible and $D \rightarrow Y$ is a near-immersion, it follows that $D \rightarrow Y$ is a minimal area disk diagram for $\partial D \rightarrow Y$. Therefore $|\eta| \leq C \cdot \Delta(|\partial D|)$, and hence the combinatorial distance between $f(a)$ and $f(b)$ in $Y \backslash\left\{f\left(x_{0}\right)\right\}$ is bounded 
by $|\eta| \leq C \cdot \Delta(\operatorname{diam}(A)+2)$. Since $a$ and $b$ were arbitrary, we have proved that inequality (5-1) holds.

Theorem 5.15 (Subgroups of 2-dimensional relatively hyperbolic groups are relatively hyperbolic) Let $Y$ be a one-connected negatively curved, fine, almost proper and cocompact $H-2-$ complex such that $H$-stabilizers of cells are slender. If $G \leq H$ is finitely presented then $G$ is hyperbolic relative to a finite collection of $G$-stabilizers of cells of $Y$.

Proof of Theorem 5.15 By Theorem 4.4, there is an equivariant tower $X \rightarrow Y$ where $X$ is a one-connected cocompact $G-2-$ complex. Since $Y$ is negatively curved and almost proper $G$-complex, the same properties hold for $X$. Proposition 5.14 implies that $X$ is fine. Proposition 5.13 implies that $G$ is hyperbolic relative to a finite collection $\mathcal{P}$ of $G$-stabilizers of cells of $X$. Since $X \rightarrow Y$ is a $G$-map, the collection $\mathcal{P}$ consists of $G$-stabilizers of cells of $Y$.

\section{References}

[1] B H Bowditch, Relatively hyperbolic groups, Internat. J. Algebra Comput. 22 (2012) 1250016, 66 MR2922380

[2] N Brady, Branched coverings of cubical complexes and subgroups of hyperbolic groups, J. London Math. Soc. 60 (1999) 461-480 MR1724853

[3] M R Bridson, A Haefliger, Metric spaces of non-positive curvature, Grundl. Math. Wissen. 319, Springer, Berlin (1999) MR1744486

[4] M R Bridson, D T Wise, $\mathscr{V} \mathscr{H}$ complexes, towers and subgroups of $F \times F$, Math. Proc. Cambridge Philos. Soc. 126 (1999) 481-497 MR1684244

[5] I M Chiswell, D J Collins, J Huebschmann, Aspherical group presentations, Math. Z. 178 (1981) 1-36 MR627092

[6] J M Corson, On finite groups acting on contractible complexes of dimension two, Geom. Dedicata 87 (2001) 161-166 MR1866846

[7] S M Gersten, Reducible diagrams and equations over groups, from: "Essays in group theory", (S M Gersten, editor), Math. Sci. Res. Inst. Publ. 8, Springer, New York (1987) 15-73 MR919828

[8] S M Gersten, Subgroups of word hyperbolic groups in dimension 2, J. London Math. Soc. 54 (1996) 261-283 MR1405055

[9] M Gromov, Hyperbolic groups, from: "Essays in group theory", (S M Gersten, editor), Math. Sci. Res. Inst. Publ. 8, Springer, New York (1987) 75-263 MR919829 
[10] F Haglund, Complexes simpliciaux hyperboliques de grande dimension, Prepublication Orsay 71 (2003)

[11] A Hatcher, Algebraic topology, Cambridge Univ. Press (2002) MR1867354

[12] J Howie, On pairs of 2-complexes and systems of equations over groups, J. Reine Angew. Math. 324 (1981) 165-174 MR614523

[13] J Howie, How to generalize one-relator group theory, from: "Combinatorial group theory and topology", (S M Gersten, J R Stallings, editors), Ann. of Math. Stud. 111, Princeton Univ. Press (1987) 53-78 MR895609

[14] G C Hruska, D T Wise, Towers, ladders and the B B Newman spelling theorem, J. Aust. Math. Soc. 71 (2001) 53-69 MR1840493

[15] T Januszkiewicz, J Świątkowski, Simplicial nonpositive curvature, Publ. Math. Inst. Hautes Études Sci. (2006) 1-85 MR2264834

[16] R Levitt, Full subcomplexes of CAT(0) simplicial complexes arXiv:1109.3502

[17] R Levitt, Group actions on CAT(0) simplicial complexes arXiv:1111.3076

[18] E Martínez-Pedroza, D T Wise, Relative quasiconvexity using fine hyperbolic graphs, Algebr. Geom. Topol. 11 (2011) 477-501 MR2783235

[19] E Martínez-Pedroza, D T Wise, Coherence and negative sectional curvature in complexes of groups, Michigan Math. J. 62 (2013) 507-536 MR3102528

[20] D Osajda, A combinatorial non-positive curvature, I: Weak systolicity arXiv: 1305.4661

[21] C D Papakyriakopoulos, On Dehn's lemma and the asphericity of knots, Ann. of Math. 66 (1957) 1-26 MR0090053

[22] J-P Serre, Trees, Springer, New York (1980) MR607504

[23] A J Sieradski, A coloring test for asphericity, Quart. J. Math. Oxford Ser. 34 (1983) 97-106 MR688427

[24] D T Wise, Sixtolic complexes and their fundamental groups, preprint (2003)

[25] D T Wise, Sectional curvature, compact cores, and local quasiconvexity, Geom. Funct. Anal. 14 (2004) 433-468 MR2062762

[26] G Zadnik, Finitely presented subgroups of systolic groups are systolic arXiv: 1307.3839

Department of Mathematics and Statistics, Memorial University of Newfoundland St. John's NL A1C 5S7, Canada

rgh262@mun.ca, emartinezped@mun.ca

www.math.mun.ca/ emartinezped/

Received: 9 July $2013 \quad$ Revised: 5 March 2014 